\title{
Maternal nicotine exposure during gestation and lactation induces kidney injury and fibrosis in rat offspring
}

\author{
Chung-Ming Chen ${ }^{1,2}$, Hsiu-Chu Chou ${ }^{3}$ and Liang-Ti Huang ${ }^{4}$
}

\begin{abstract}
BACKGROUND: Maternal tobacco smoke exposure adversely affected fetal kidney development. Nicotine stimulates epithelial-mesenchymal transition and connective tissue growth factor (CTGF) expression in the renal epithelium. We hypothesized that maternal nicotine exposure would induce kidney fibrosis and involve CTGF in newborn rats.
\end{abstract}

METHODS: Nicotine was administered to pregnant SpragueDawley rats at a dose of $6 \mathrm{mg} / \mathrm{kg} / \mathrm{d}$ from gestational days $7-21$ and gestational day 7 to postnatal day 14 . A control group was injected with normal saline. Neonatal kidney tissues underwent histological analysis, collagen measurement, and western blot analysis.

RESULTS: Tubular injury scores and total collagen contents were significantly higher in rats born to nicotine-treated dams than in rats born to normal saline-treated dams on postnatal days 7 and 21. Masson's trichrome staining further verified the presence of kidney fibrosis. Prenatal and/or postnatal nicotine exposure increased CTGF expression on postnatal days 7 and 21 .

CONCLUSION: Maternal nicotine exposure during gestation and lactation induces neonatal kidney fibrosis, and CTGF may be involved in the pathogenesis of kidney fibrosis. These results may be relevant to premature low-birth-weight infants who are conveyed a high risk of developing chronic kidney disease and exposed to breast milk of smoking mothers during the neonatal period.

M aternal smoking during pregnancy has been associated with fetal growth retardation, premature delivery, and profoundly affects development of multiple organ systems (1). Nicotine is the causative agent for these effects because it is a major pharmacological constituent of tobacco smoke that readily crosses the placenta and is concentrated in the fetus 15 percent higher than maternal level (2). Human and animal studies have demonstrated that maternal nicotine and tobacco smoke exposure adversely affected fetal kidney development. These are manifested by kidney malformations, decreased kidney weight and volume, and glomerular mass and elevated blood pressure in the offspring (3-7). The exact mechanism by which maternal nicotine impairs kidney growth in offspring remains unknown, but impaired kidney growth is linked to hypertension (8).

Nicotine has toxic effects in the mesangial cells and proximal tubules of the kidney and causes renal damage $(9,10)$. Nicotine also stimulates expression of markers of epithelialmesenchymal transition, such as vimentin, fibronectin, and $\alpha$-smooth muscle actin in the renal epithelium $(9,10)$. Because nicotine easily crosses the placenta, entering fetal blood, and is distributed in breast milk, the fetal and neonatal rats would be expected to receive nicotine via the placenta and mother's milk $(11,12)$. Taken together, these studies indicate that maternal nicotine exposure may induce kidney fibrosis in the offspring.

Connective tissue growth factor (CTGF) is part of the CTGFCyr61/Cef10-Nov family (13). CTGF plays a crucial role in kidney pathogenesis, which include migration, hypertrophy, and fibronectin production in mesangial cells, epithelialmesenchymal transition and fibronectin production of tubular epithelial cells, and collagen production by renal interstitial fibroblasts (14-18). Nicotine has been reported to increase CTGF mRNA and protein expression in proximal tubular epithelial cells and human gingival fibroblasts $(19,20)$. There are limited data of the effects of maternal nicotine exposure during gestation and lactation on kidney morphometry and fibrosis in rat offspring. We hypothesized that maternal nicotine exposure would induce kidney injury and fibrosis in rat offspring. The aims of our study were to determine the effects of maternal nicotine exposure on kidney histology and fibrosis and to elucidate the relationship between CTGF and collagen expression in maternal nicotine-induced kidney fibrosis in rats.

\section{RESULTS}

There were 23 pups of 3 dams in the normal saline-treated group, 25 pups of 3 dams in the prenatal nicotine-treated group, and 24 pups of 3 dams in the prenatal and postnatal nicotine-treated group. No significant difference in litter size existed among study groups. 
Prenatal Nicotine Effects on Maternal Body Weight

The maternal body weight before treatment and total body weight gain were comparable among normal saline-treated and two nicotine-treated groups from gestational days 7-19.

\section{Body Weight, Kidney Weight, and Kidney to Body Weight Ratios}

Maternal nicotine administration had no significant effect on body weights and kidney weights on postnatal days 7 and 21 (Tables 1 and 2). The kidney/body weight ratios were comparable among rats born to normal saline-treated and nicotinetreated dams on postnatal day 7 . On postnatal day 21 , rats born to prenatal and postnatal nicotine-treated dams exhibited significantly lower kidney/body weight ratios than the rats born to normal saline-treated dams.

\section{Histology Results}

Representative kidney sections stained with hematoxylin and eosin from maternal normal saline-exposed and

Table 1. Body weights, kidney weights, and the kidney/body weight ratios in rat pups born to normal saline- or nicotine-treated mothers on postnatal day 7

\begin{tabular}{lcccc}
\hline Maternal treatment & $n$ & $\begin{array}{c}\text { Body } \\
\text { weight }(\mathrm{g})\end{array}$ & $\begin{array}{c}\text { Kidney } \\
\text { weight }(\mathrm{g})\end{array}$ & $\begin{array}{c}\text { Kidney/body } \\
\text { weight (\%) }\end{array}$ \\
\hline Saline & 12 & $13.89 \pm 2.89$ & $0.20 \pm 0.04$ & $1.41 \pm 0.09$ \\
2Nicotine & 13 & $14.80 \pm 0.47$ & $0.19 \pm 0.01$ & $1.32 \pm 0.08$ \\
4Nicotine & 12 & $14.81 \pm 0.91$ & $0.21 \pm 0.01$ & $1.43 \pm 0.09$ \\
\hline
\end{tabular}

Values represent means \pm SD. nicotine-exposed rats on postnatal days 7 and 21 are presented in Figures 1a and 2a. Tubular atrophy, dilatation of the tubular lumen, vacuolar degeneration of the tubular epithelia, increased space between the renal tubules, and inflammatory cells infiltration were observed in the maternal nicotine-treated groups (Figure 1a). Tubular injury was not seen in the rats born to normal saline-treated dams. Tubular injury scores were significantly higher in the rats born to nicotine-treated dams than in rats born to normal saline-treated dams on postnatal days 7 and 21 (Figure 1b,c). Rats born to prenatal and postnatal nicotine-treated dams displayed significantly smaller mean glomerular size compared with rats born to normal saline-treated dams on postnatal day 7 (Figure $\left.2 \mathbf{b},{ }^{\star} P<0.05\right)$. Mean glomerular sizes were comparable among rats born to normal saline- and nicotine-treated dams on postnatal day 21 .

Table 2. Body weights, kidney weights, and the kidney/body weight ratios in rat pups born to normal saline- or nicotine-treated mothers on postnatal day 21

\begin{tabular}{lcccc} 
Maternal treatment & $n$ & $\begin{array}{c}\text { Body } \\
\text { weight (g) }\end{array}$ & $\begin{array}{c}\text { Kidney } \\
\text { weight (g) }\end{array}$ & $\begin{array}{c}\text { Kidney/body } \\
\text { weight (\%) }\end{array}$ \\
\hline Saline & 11 & $43.00 \pm 4.88$ & $0.54 \pm 0.05$ & $1.27 \pm 0.07$ \\
2Nicotine & 12 & $45.47 \pm 3.92$ & $0.55 \pm 0.05$ & $1.21 \pm 0.07$ \\
4Nicotine & 12 & $44.46 \pm 2.29$ & $0.52 \pm 0.04$ & $1.18 \pm 0.06^{*}$
\end{tabular}

Values represent means $\pm \mathrm{SD}$.

*Different from maternal normal saline-treated rats at $P<0.01$. a

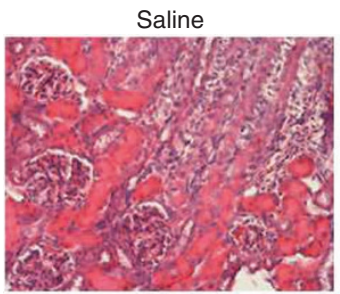

$21 \mathrm{~d}$

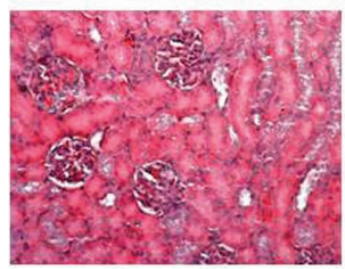

b

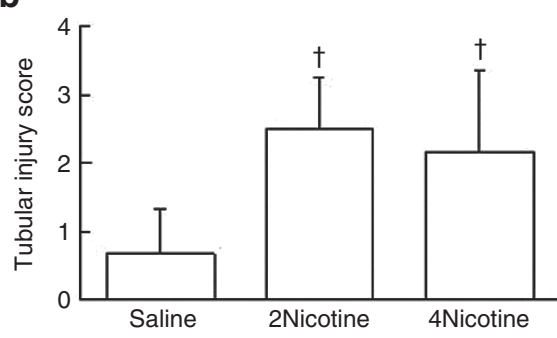

2Nicotine
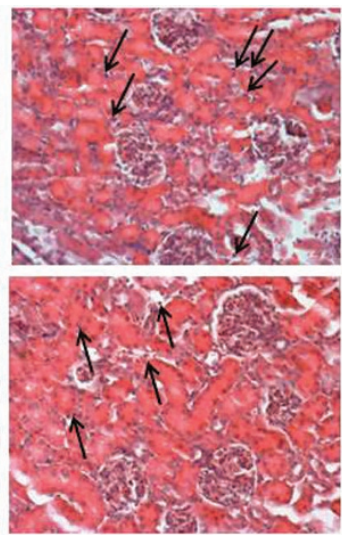

C

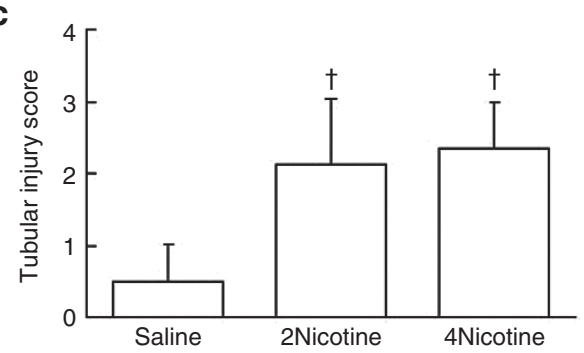

Figure 1. Histology and tubular injury score in kidney tissues on postnatal days 7 and 21. (a) Representative photomicrographs of kidney and tubular injury scores in maternal normal saline- or nicotine-exposed rats on postnatal days (b) 7 and (c) 21. (a) Inflammatory cells (arrow) in the interstitial area and tubular atrophy and vacuolar degeneration in the tubule epithelia were found in the rats born to nicotine-treated dams. (b,c) Tubular injury scores were significantly higher in the rats born to maternal nicotine-treated dams on postnatal days 7 and $21\left({ }^{+} P<0.001\right)$. Saline $=$ maternal saline treatment from gestational days 7-21 $(n=11-12)$, 2Nicotine $=$ maternal nicotine treatment from gestational days $7-21(n=12-13), 4$ Nicotine $=$ maternal nicotine treatment from gestational day 7 to postnatal day $14(n=12)$. Bar $=100 \mu \mathrm{m}$. 


\section{Articles $\mid$ Chen et al.}

a

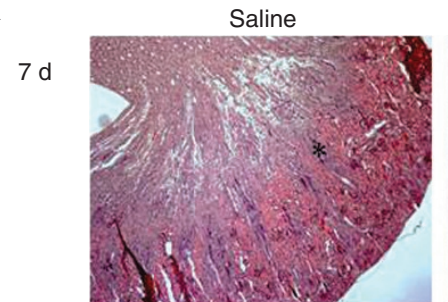

$21 \mathrm{~d}$

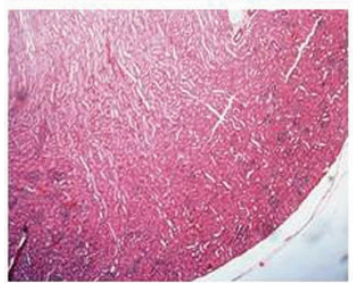

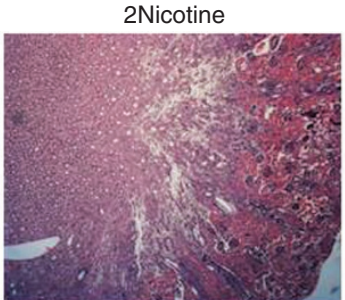

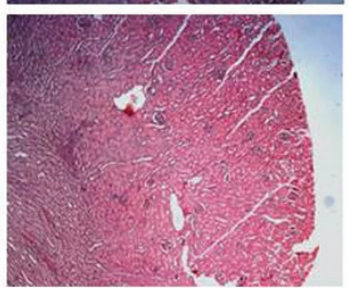

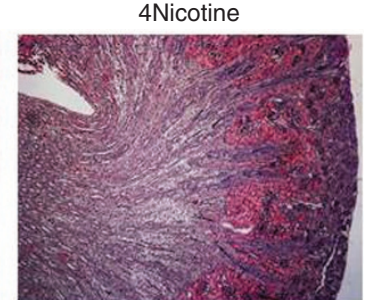

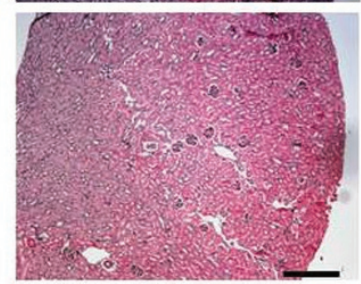

b

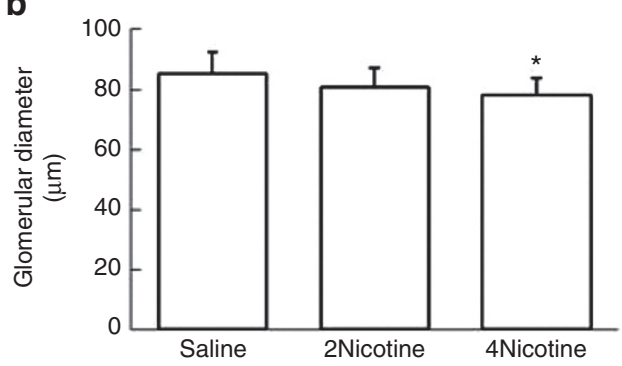

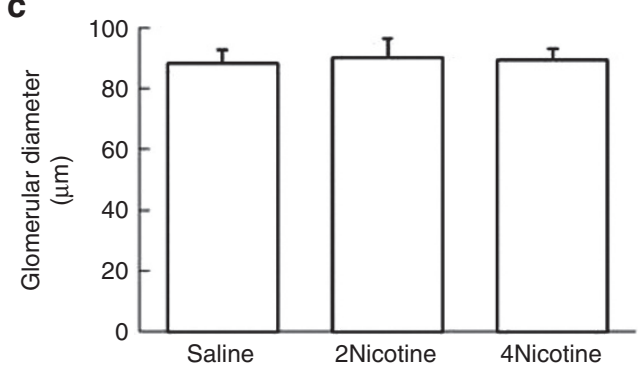

Figure 2. Histology and mean glomerular size in kidney tissues on postnatal days 7 and 21. (a) Representative photomicrographs of kidney and mean glomerular size in maternal normal saline- or nicotine-exposed rats on postnatal days (b) 7 and (c) 21 . Rats born to 4 Nicotine-treated dams displayed significantly smaller mean glomerular size compared with rats born to normal saline-treated dams on postnatal day $7\left({ }^{*} P<0.05\right)$. Saline $=$ maternal saline treatment from gestational days 7-21 $(n=11-12)$, 2Nicotine $=$ maternal nicotine treatment from gestational days $7-21(n=12-13), 4$ Nicotine $=$ maternal nicotine treatment from gestational day 7 to postnatal day $14(n=12)$. Bar $=600 \mu \mathrm{m}$.

\section{Total Collagen Content}

Total collagen content in kidney tissues increased as the rats aged (Figure 3a,b). The rats born to nicotine-treated dams had significantly higher total collagen contents compared with rats born to normal saline-treated dams on postnatal day 7 (Figure 3a). On postnatal day 21 , total collagen contents were significantly higher in the rats born to prenatal and postnatal nicotine-treated dams compared to rats born to normal saline treated-treated dams (Figure 3b). Masson's trichrome staining further verified the presence of kidney fibrosis (Figure 3c). Rats born to nicotine-treated dams exhibited increased collagen deposition in the glomerular mesangial matrix and tubular interstitium compared with rats born to normal saline-treated dams.

\section{Western Blot Analysis of CTGF}

The rats born to nicotine-treated dams had significantly higher CTGF protein expression in kidney tissues than the rats born to normal saline-treated dams on postnatal days 7 and 21 (Figure 4).

\section{Immunohistochemistry for CTGF}

The CTGF immunoreactivity was primarily detected in the glomeruli and in some tubular cells (Figure 5a). Rats born to nicotine-treated dams displayed significantly higher optical density of CTGF compared with rats born to normal salinetreated dams on postnatal days 7 and 21 (Figure 5b,c).

\section{DISCUSSION}

Our in vivo model showed that prenatal and postnatal nicotine exposure injured renal tubules and increased total collagen content in the kidneys during the first postnatal week, as confirmed by Masson's trichrome staining in kidney tissues. Prolonged exposure to nicotine increased total collagen content and caused kidney fibrosis in the third postnatal week. The major findings of our study are that prenatal and postnatal exposure to nicotine-induced kidney injury and caused kidney fibrosis in the rat offspring. The nicotine effect on kidney fibrosis was associated with the increased expression of CTGF protein. These results suggest that CTGF is involved in the pathogenesis of kidney injury and fibrosis induced by nicotine exposure.

Cigarette smoke contains over 4,000 chemicals, including arsenic, hydrogen cyanide, lead, nicotine, and tar that may be toxic to the fetus (21). However, data from animal studies suggest that fetal and neonatal exposure to nicotine alone may result in postnatal metabolic alterations associated with obesity, type 2 diabetes, hypertension, and hypertriglyceridemia (22). These results suggest that nicotine is the single most important component of cigarette smoke that causes adverse health outcomes in the offspring.

In the present study, we found that prenatal and postnatal nicotine administration had no significant effect on body weights and kidney weights on postnatal days 7 and 21 . The 
a

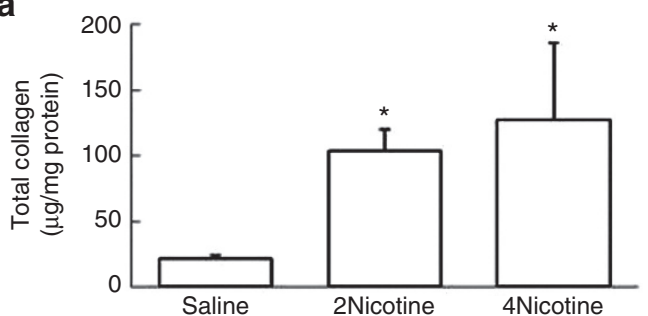

b

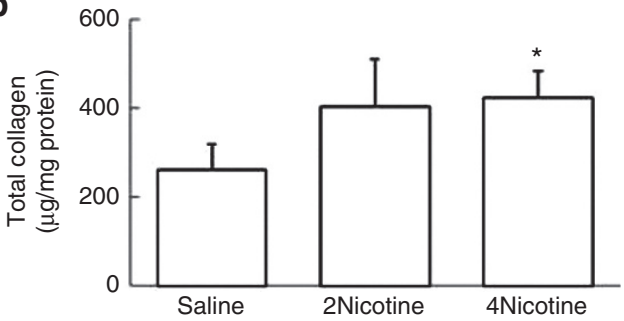

C
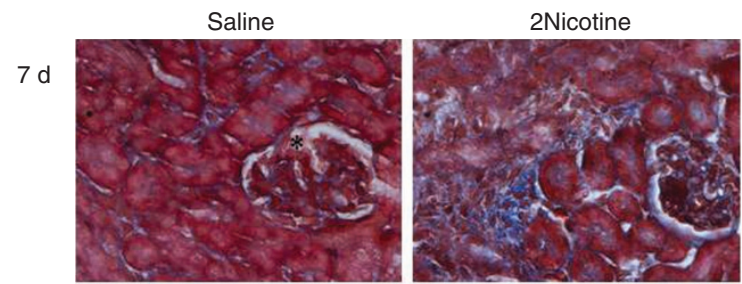

$21 \mathrm{~d}$
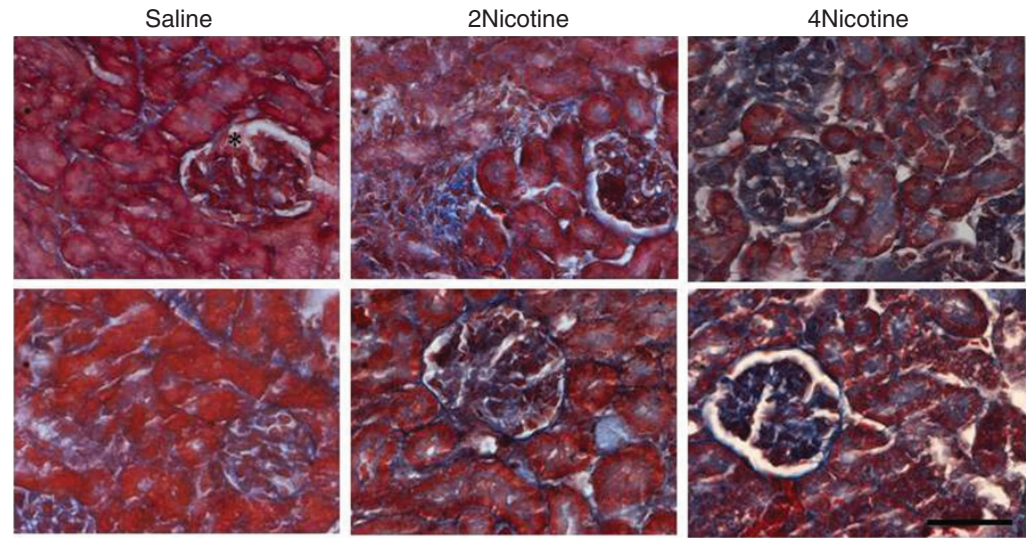

Figure 3. Total collagen content and Masson's trichrome staining in kidney tissues on postnatal days 7 and 21 . Total collagen content in kidney tissues on postnatal days 7 (a) and 21 (b), and Masson's trichrome staining (c). (a,b) Total collagen contents were significantly higher in the rats born to prenatal and postnatal nicotine-treated dams compared to rats born to normal saline treated-treated dams on postnatal days 7 and $21(* P<0.05)$. (c) Rats born to nicotine-treated dams had widespread collagen deposition in both glomeruli and interstitium compared with rats born to normal saline-treated dams. Saline $=$ maternal saline treatment from gestational days 7 to $21(n=5)$, 2Nicotine = maternal nicotine treatment from gestational days 7-21 $(n=5)$, 4Nicotine = maternal nicotine treatment from gestational day 7 to postnatal day $14(n=5)$. Bar $=100 \mu \mathrm{m}$.

a

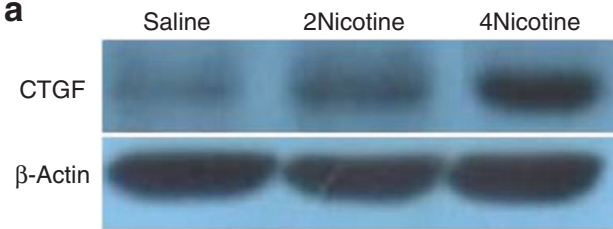

C

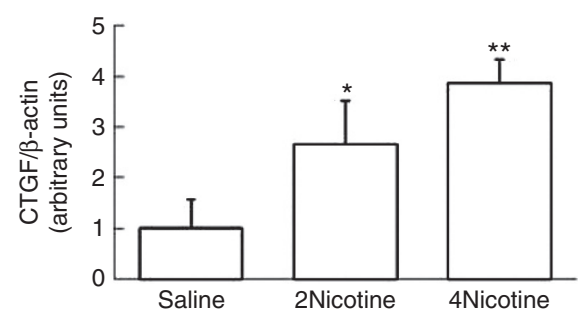

b

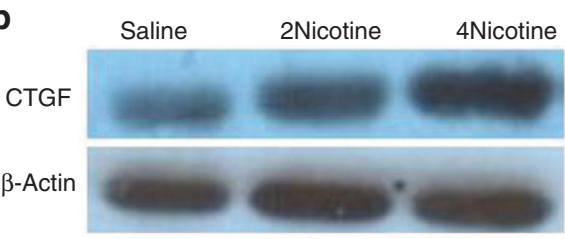

d

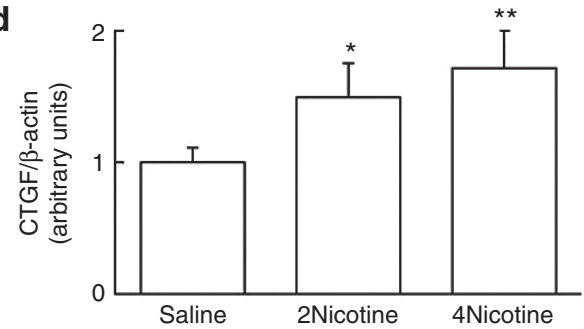

Figure 4. Representative western blotting and scanning densitometry results of the connective tissue growth factor (CTGF) protein in kidney tissues on postnatal days 7 and 21. The expression of the CTGF protein was higher in the rats born to maternal nicotine-treated dams on postnatal days 7 (a) and 21 (b). The protein expression levels were normalized to $\beta$-actin for each animal. Data were reported as the fold-change relative to control rat pups. The level of CTGF expression was significantly higher in rats born to nicotine-treated dams compared with rats born to normal saline-treated dams on postnatal days 7 (c) and $21(\mathbf{d})\left({ }^{*} P<0.05,{ }^{* *} P<0.01\right)$. Saline = maternal saline treatment from gestational days $7-21(n=4), 2$ Nicotine $=$ maternal nicotine treatment from gestational days 7-21 $(n=4)$, 4Nicotine = maternal nicotine treatment from gestational day 7 to postnatal day $14(n=4)$.

kidney/body weight ratios were comparable among rats on postnatal day 7. These results were different to those of Mao et al. (23) who found that maternal nicotine administration during pregnancy resulted in significantly decreased kidney weight and kidney to body weight ratio on postnatal day 14 . We speculated that the discrepancy was due to different gestational exposure times. In rats born to prenatal and postnatal nicotine-treated dams, loss of kidney weight was proportionally greater than body weight loss, resulting in a decreased kidney to body weight ratio on postnatal day 21 . These results suggest that the effect of maternal smoking on kidney growth is proportional to the duration of nicotine exposure. The underlying mechanisms are not known. Disruption of cell cycle and renin-angiotensin system may be involved in the 


\section{Articles $\mid$ Chen et al.}

a
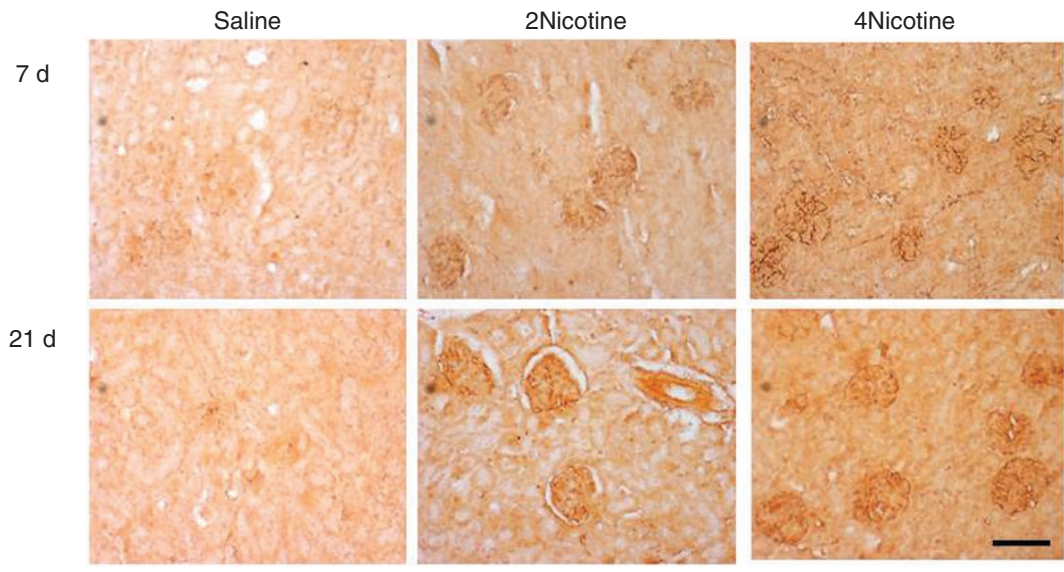

b

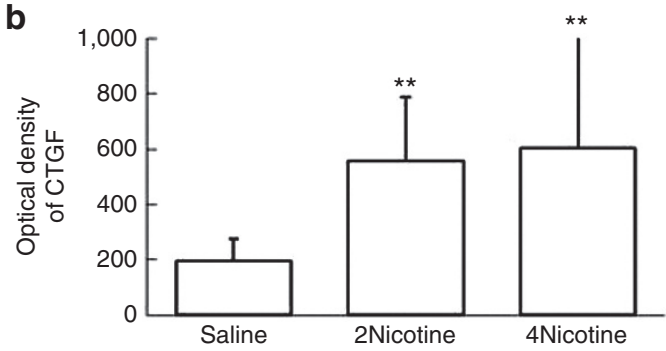

C

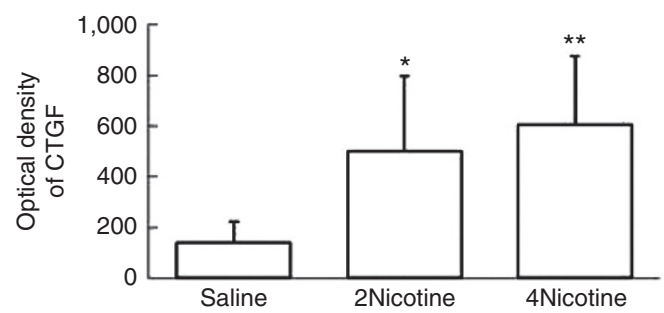

Figure 5. Representative photomicrographs and optical density of connective tissue growth factor (CTGF) in maternal normal saline- or nicotine-exposed rats on postnatal days 7 and 21. (a) The CTGF immunoreactivity was primarily localized in the glomeruli and in some tubular cells and was enhanced in the kidney tissues of rats born to nicotine-treated dams on postnatal days 7 and 21 . Positive immunoreactivity is indicated by brown staining. Rats born to nicotine-treated dams displayed significantly higher optical density of CTGF compared with rats born to normal saline-treated dams on postnatal days 7 (b) and 21 (c) $\left({ }^{*} P<0.05,{ }^{* *} P<0.01\right)$. Saline $=$ maternal saline treatment from gestational days $7-21(n=11-12), 2$ Nicotine $=$ maternal nicotine treatment from gestational days $7-21(n=12-13)$, 4Nicotine = maternal nicotine treatment from gestational day 7 to postnatal day 14 $(n=12)$. Bar $=160 \mu \mathrm{m}$.

pathogenesis because maternal exposure to cigarette smoke/ nicotine decreased cell proliferation, increased apoptosis of renal cells, and reduced renal AT2 receptor expression $(24,25)$.

After embryogenesis, the mammalian kidney develops in three successive phases: the pronephros, mesonephros, and metanephros phases. Nephrogenesis is complete by the 36th week of gestation in humans, but continues until approximately postnatal day 10 in rats (26). In this study, newborn rats were exposed to nicotine during the immediate postnatal period. We found that rats exposed to prenatal and postnatal nicotine exhibited inflammatory cells infiltration and higher grade of tubular injuries and smaller glomeruli than did rats expose to normal saline. These results are consistent to those of Sánchez-López et al. (27) who found that CTGF recruits inflammatory cells and induces fibrosis in the kidney of mice. Acute kidney injury is common in neonatal intensive care units $(28,29)$ and premature low birth weight conveyed a higher risk of developing chronic kidney injury compared to normal birth weight infants $(30,31)$. These results suggest that maternal smoking during gestation and/or lactation may exacerbate acute kidney injury-induced tubulointerstitial injury and progression to chronic kidney disease.

In this study, we found rats exposed to prenatal and postnatal nicotine displayed significantly smaller mean glomerular size compared with rats exposed to normal saline on postnatal day 7. This effect was less obvious on postnatal day 21. The effect of nicotine on kidney structure was related to the dosage and duration of nicotine exposure and the offspring age $(5,7,8)$. Gao et al. (5) injected nicotine $(1 \mathrm{mg} / \mathrm{kg} /$ day $)$ to female WKY rats 2 wk before mating until weaning and found comparable kidney weight, total number of nephrons, and glomerular size in rat offspring at the age of $26 \mathrm{wk}$. Toledo-Rodriguez et al. (7) continuously infused nicotine $(6 \mathrm{mg} / \mathrm{kg} /$ day $)$ to spontaneously hypertensive rats (SHR) rats throughout gestation and found decreased glomerular mass in SHR offspring at the $9 \mathrm{wk}$ of age. Zarzeck et al. applied cigarette-smoke condensate containing $1 \mathrm{mg}$ of nicotine to oral mucosa of pregnant Sprague-Dawley rat twice-daily and found lower glomerular volume at $12 \mathrm{wk}$ of age. Extrapolating data from the rodent model to humans must be treated with caution; however, certain clinical implications are yielded by the current findings.

Fibrosis is a common response to different kidney injuries and is characterized by the disproportionate accumulation of extracellular matrices that replace the normally functioning parenchyma (32). Kidney fibrosis is a complex condition and its underlying mechanisms remain largely unknown. Current concept suggests that abnormal wound healing of the kidney tissue following recurrent injury is the main cause of the fibrotic response. CTGF is a fibroblast mitogen and promoter of collagen deposition that acts downstream of transforming growth factor- $\beta 1$, particularly regarding its profibrotic effects $(18,33,34)$. In this study, we found that nicotine exposure during gestation and lactation increased total collagen contents 
and CTGF protein expression levels in kidney tissues on postnatal days 7 and 21. These results suggest that maternal nicotine-induced kidney fibrosis is regulated by CTGF.

The limitation of this study is that we do not distinguish rat pups to male and females. The effects of gender on kidney injury and fibrosis following maternal nicotine exposure are unknown. Mao et al. found that maternal nicotine administration during pregnancy resulted in comparable decrease in kidney weight and kidney/body weight ratio between male and female offspring of 14-d-old and 30-d-old. Dündar et al. (35) reported similar glomerular size in both male and female healthy rats exposed to passive smoking after birth. These studies suggest no gender effects of maternal nicotine on kidney structure during the immediate postnatal period in rats.

In conclusion, we showed that prenatal and postnatal nicotine exposure increased total collagen contents in the kidneys of rats during the postnatal weeks. The development of maternal nicotine-induced kidney fibrosis was associated with increased CTGF expression in rat offspring. These results suggest that CTGF may be involved in the pathogenesis of kidney fibrosis induced by nicotine exposure. Further investigation, both in vitro and in vivo, is needed to evaluate the role of CTGF may provide insights into new preventive strategies in maternal nicotine-induced kidney fibrosis. Overall, these results suggest that neonatal kidney are sensitive to cigarette smoke, which may induce the development of kidney fibrosis, and efforts should be made to discourage women from smoking during pregnancy and postnatal period.

\section{METHODS}

\section{Animals}

The Animal Care and Use Committee of Taipei Medical University approved this study, which was performed with timed pregnant Sprague-Dawley rats. The kidney tissues used for these experiments were obtained from a previous study designed to assess lung fibrosis (36). All rats described in the present manuscript were kept at room air and were not exposed to $95 \% / 60 \% \mathrm{O}_{2}$.

\section{Maternal Nicotine Administration}

Nicotine tartrate (Sigma, St Louis, MO) was administrated through osmotic mini-pump implanted subcutaneously. On the gestational day 7 of pregnancy, rats were anesthetized with pentobarbital, and an incision was made on the back to insert osmotic mini-pumps (Figure 6, 2ML2 and 2ML4; Alzet, Cupertino, CA). The incision was closed with three sutures. Six pregnant rats were implanted with mini-pumps containing nicotine at a concentration of $40 \mu \mathrm{g} / \mu \mathrm{l}$, and the other three were implanted with mini-pumps containing only sterile normal saline, which served as the vehicle control. The minipump delivered nicotine at a dose of $6 \mathrm{mg} / \mathrm{kg} /$ day with blood concentrations closely resembles those occurring in moderate to heavy human smokers $(37,38)$. According to the manufacturer's specifications, the delivery periods for the mini-pumps are 14 (2ML2) and 28 (2ML4) days; so, nicotine delivery continued at birth (2Nicotine) and after birth until postnatal day 14 (4Nicotine), respectively. Dams are housed in individual cages with free access to laboratory food and water ad libitum, kept on a 12:12-h light-dark cycle, and allowed to deliver vaginally at term. Maternal nicotine treatment did not affect the length of gestation.

\section{Experimental Protocol}

Within $12 \mathrm{~h}$ of birth, litters from each treatment were pooled and randomly redistributed to the newly delivered mothers to eliminate litter differences and to equalize the number of runts in each group. Pups were euthanized by intraperitoneal injections of pentobarbital

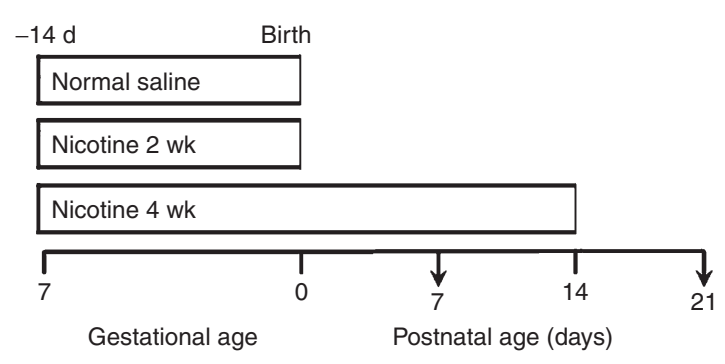

Figure 6. Diagrammatic representation of the experimental design showing the study timeline and the rat pup treatment groups. $\downarrow$ indicates time of sacrifice.

$(100 \mathrm{mg} / \mathrm{kg})$ on postnatal days 7 and 21 , and body and kidney weights were recorded. One kidney per animal was used for histological analysis and the second one was used for total collagen measurement and western blot analysis.

\section{Measurement of Total Collagen}

Total collagen was determined by assaying total soluble collagen using the Sircol collagen assay kit (Biocolor, Newton Abbey, UK) as previously reported (39). Briefly, kidney tissues were homogenized in $5 \mathrm{ml}$ of $0.5 \mathrm{~mol} / \mathrm{l}$ acetic acid containing $1 \mathrm{mg}$ of pepsin (Sigma Chemical, St Louis, MO) per $10 \mathrm{mg}$ tissue residue. Each sample was incubated for $24 \mathrm{~h}$ at $4{ }^{\circ} \mathrm{C}$ with stirring. After centrifugation, $100 \mu \mathrm{l}$ of each supernatant was assayed. One milliliter of Sircol dye reagent that specifically binds to collagen was then added to each sample and mixed for $30 \mathrm{~min}$. After centrifugation, the pellet was suspended in $1 \mathrm{ml}$ of alkali reagent $(0.5 \mathrm{~mol} / \mathrm{l} \mathrm{NaOH})$ included in the kit, and the optical density was evaluated at $540 \mathrm{~nm}$ with a spectrophotometer.

\section{Histological Examination}

The kidney was placed in $4 \%$ paraformaldehyde and washed in phosphate-buffered saline and serially dehydrated in increasing concentrations of ethanol before being embedded in paraffin. Sevenmicrometer tissue sections were stained with hematoxylin and eosin or Masson's trichrome, examined by light microscopy, and assessed for the kidney morphology and fibrosis. The histological analysis of the kidney was modified according to suggestions in ToledoRodriguez et al. (7). The sizes of the individual glomeruli located in the middle cortex and juxtamedullary zone were calculated as the average of the largest and smallest glomerular diameters within a field of view; the calculations involved $10 \pm 5$ glomeruli per kidney. Kuruş et al. (40) defined tubular injury as tubular dilation, tubular atrophy, vacuolization, the degeneration and sloughing of tubular epithelial cells, or thickening of the tubular basement membrane. Only cortical tubules were used in the scoring system, where $0=$ no tubular injury; $1=<10 \%$ of tubules injured; $2=10 \%-25 \%$ of tubules injured; $3=26 \%-50 \%$ of tubules injured; $4=51 \%-75 \%$ of tubules injured; and $5=>75 \%$ of tubules injured.

\section{Western Blot Analysis}

Kidney tissues were homogenized in ice-cold buffer containing 50 $\mathrm{mmol} / \mathrm{l}$ of Tris. $\mathrm{HCl}$ ( $\mathrm{pH} 7.5), 1 \mathrm{mmol} / \mathrm{l}$ of ethylene glycol tetraacetic acid, $1 \mathrm{mmol} / \mathrm{l}$ of ethylenediaminetetraacetic acid, and a protease inhibitor cocktail (complete mini-tablets; Roche, Mannheim, Germany). Proteins $(30 \mu \mathrm{g})$ were resolved on $12 \%$ sodium dodecyl sulfate-polyacrylamide gel electrophoresis under reducing conditions and electroblotted to a polyvinylidene difluoride membrane (Immobilon $^{\mathrm{P}}$, Millipore, Bedford, MA). After being blocked with $5 \%$ non-fat dry milk, the membranes were incubated with antiCTGF $(1: 2,000$, Abcam, Cambridge, UK) or anti- $\beta$-actin $(1: 20,000$, Sigma-Aldrich, St Louis, MO), and then incubated with horseradish peroxidase-conjugated goat anti-mouse IgG (Pierce Biotechnology, Rockford, IL). Protein bands are detected using SuperSignal Substrate from Pierce. Densitometric analysis was performed to measure the intensity of CTGF and $\beta$-actin bands using AIDA software. The densitometry unit of the protein expression in the normal saline-treated group was assigned as 1 after normalized with $\beta$-actin. 
Immunohistochemistry and Semiquantitative Analysis for CTGF Immunostaining was performed on $7-\mu \mathrm{m}$ thick kidney paraffin sections with immunoperoxidase visualization. After blocking endogenous peroxidase activity and nonspecific binding of antibody, sections were first preincubated for $1 \mathrm{~h}$ at room temperature in $0.1 \mathrm{~mol} / \mathrm{l}$ phosphate-buffered saline containing $10 \%$ normal goat serum and $0.3 \% \mathrm{H}_{2} \mathrm{O}_{2}$ before being incubated for $20 \mathrm{~h}$ at $4{ }^{\circ} \mathrm{C}$ with goat polyclonal anti-CTGF (L-20) antibodies (1:50 dilutions; Santa Cruz Biotechnology, Santa Cruz, CA) was used as primary antibodies. The sections were then treated for $1 \mathrm{~h}$ at room temperature with biotinylated rabbit anti-goat IgG (1:200, Jackson ImmunoResesarch Labotories, West Grove, PA). Followed by reaction with the reagents from an $\mathrm{ABC}$ kit (Avidin-Biotin Complex, Vector, $\mathrm{CA}$ ) as the manufacturer's recommendations, the reaction products were visualized by incubating with diaminobenzidine. All immunostained sections were viewed and photographed by Nikon Eclipse E600. During semiquantitative analysis, the optical density values of the positive cells showed brown articles or clumps in the cytoplasm. The positive staining area in the glomeruli and tubules under $\times 400$ field of each section was assessed by using Image Pro Plus 6.0 (Media Cybernetics, Silver Spring).

\section{Statistical Analysis}

Data are presented as the mean \pm SD. Analysis of difference among multiple groups was carried out by one-way ANOVA, and significance is determined using Bonferroni's correction for multiple comparisons. Differences are considered significant at $P<0.05$.

\section{STATEMENT OF FINANCIAL SUPPORT}

No financial assistance was received to support this study.

Disclosure: The authors declare no conflict of interest.

\section{REFERENCES}

1. Abbott LC, Winzer-Serhan UH. Smoking during pregnancy: lessons learned from epidemiological studies and experimental studies using animal models. Crit Rev Toxicol 2012;42:279-303.

2. Rehan VK, Wang Y, Sugano S, et al. Mechanism of nicotine-induced pulmonary fibroblast transdifferentiation. Am J Physiol Lung Cell Mol Physiol 2005;289:L667-76.

3. Källen K. Maternal smoking and urinary organ malformations. Int J Epidemiol 1997;26:571-4.

4. Pausová Z, Paus T, Sedová L, Bérubé J. Prenatal exposure to nicotine modifies kidney weight and blood pressure in genetically susceptible rats: a case of gene-environment interaction. Kidney Int 2003;64:829-35.

5. Gao YJ, Holloway AC, Su LY, Takemori K, Lu C, Lee RM. Effects of fetal and neonatal exposure to nicotine on blood pressure and perivascular adipose tissue function in adult life. Eur J Pharmacol 2008;590:264-8.

6. Taal HR, Geelhoed JJ, Steegers EA, et al. Maternal smoking during pregnancy and kidney volume in the offspring: the Generation R Study. Pediatr Nephrol 2011;26:1275-83.

7. Toledo-Rodriguez M, Loyse N, Bourdon C, Arab S, Pausova Z. Effect of prenatal exposure to nicotine on kidney glomerular mass and AT1R expression in genetically diverse strains of rats. Toxicol Lett 2012;213: $228-34$.

8. Zarzecki M, Adamczak M, Wystrychowski A, Gross ML, Ritz E, Wiecek A. Exposure of pregnant rats to cigarette-smoke condensate causes glomerular abnormalities in offspring. Kidney Blood Press Res 2012;36:162-71.

9. Jaimes EA, Tian RX, Raij L. Nicotine: the link between cigarette smoking and the progression of renal injury? Am J Physiol Heart Circ Physiol 2007;292:H76-82.

10. Arany I, Reed DK, Grifoni SC, Chandrashekar K, Booz GW, Juncos LA. A novel U-STAT3-dependent mechanism mediates the deleterious effects of chronic nicotine exposure on renal injury. Am J Physiol Renal Physiol 2012;302:F722-9.

11. Luck W, Nau H. Nicotine and cotinine concentrations in serum and urine of infants exposed via passive smoking or milk from smoking mothers. J Pediatr 1985;107:816-20.
12. Dahlström A, Lundell B, Curvall M, Thapper L. Nicotine and cotinine concentrations in the nursing mother and her infant. Acta Paediatr Scand 1990;79:142-7.

13. Bradham DM, Igarashi A, Potter RL, Grotendorst GR. Connective tissue growth factor: a cysteine-rich mitogen secreted by human vascular endothelial cells is related to the SRC-induced immediate early gene product CEF-10. J Cell Biol 1991;114:1285-94.

14. Abdel-Wahab N, Weston BS, Roberts T, Mason RM. Connective tissue growth factor and regulation of the mesangial cell cycle: role in cellular hypertrophy. J Am Soc Nephrol 2002;13:2437-45.

15. Burns WC, Twigg SM, Forbes JM, et al. Connective tissue growth factor plays an important role in advanced glycation end product-induced tubular epithelial-to-mesenchymal transition: implications for diabetic renal disease. J Am Soc Nephrol 2006;17:2484-94.

16. Nagae T, Mori K, Mukoyama M, et al. Adrenomedullin inhibits connective tissue growth factor expression, extracellular signal-regulated kinase activation and renal fibrosis. Kidney Int 2008;74:70-80.

17. Phanish MK, Winn SK, Dockrell ME. Connective tissue growth factor(CTGF, CCN2)-a marker, mediator and therapeutic target for renal fibrosis. Nephron Exp Nephrol 2010;114:e83-92.

18. Mason RM. Fell-Muir lecture: Connective tissue growth factor (CCN2) - a pernicious and pleiotropic player in the development of kidney fibrosis. Int J Exp Pathol 2013;94:1-16.

19. Khanna AK, Xu J, Baquet C, Mehra MR. Adverse effects of nicotine and immunosuppression on proximal tubular epithelial cell viability, tissue repair and oxidative stress gene expression. J Heart Lung Transplant 2009;28:612-20.

20. Takeuchi H, Kubota S, Murakashi E, et al. Nicotine-induced CCN2: from smoking to periodontal fibrosis. J Dent Res 2010;89:34-9.

21. Swauger JE, Steichen TJ, Murphy PA, Kinsler S. An analysis of the mainstream smoke chemistry of samples of the U.S. cigarette market acquired between 1995 and 2000. Regul Toxicol Pharmacol 2002;35 (2 Pt 1):142-56.

22. Ma N, Nicholson CJ, Wong M, Holloway AC, Hardy DB. Fetal and neonatal exposure to nicotine leads to augmented hepatic and circulating triglycerides in adult male offspring due to increased expression of fatty acid synthase. Toxicol Appl Pharmacol 2014;275:1-11.

23. Mao C, Wu J, Xiao D, et al. The effect of fetal and neonatal nicotine exposure on renal development of AT(1) and AT(2) receptors. Reprod Toxicol 2009;27:149-54.

24. Nelson E, Goubet-Wiemers C, Guo Y, Jodscheit K. Maternal passive smoking during pregnancy and foetal developmental toxicity. Part 2: histological changes. Hum Exp Toxicol 1999;18:257-64.

25. Guron G, Friberg P. An intact renin-angiotensin system is a prerequisite for normal renal development. J Hypertens 2000;18:123-37.

26. Márquez MG, Cabrera I, Serrano DJ, Sterin-Speziale N. Cell proliferation and morphometric changes in the rat kidney during postnatal development. Anat Embryol 2002;205:431-40.

27. Sánchez-López E, Rayego S, Rodrigues-Díez R, et al. CTGF promotes inflammatory cell infiltration of the renal interstitium by activating NFkappaB. J Am Soc Nephrol 2009;20:1513-26.

28. Koralkar R, Ambalavanan N, Levitan EB, McGwin G, Goldstein S, Askenazi $\mathrm{D}$. Acute kidney injury reduces survival in very low birth weight infants. Pediatr Res 2011;69:354-8.

29. Selewski DT, Jordan BK, Askenazi DJ, Dechert RE, Sarkar S. Acute kidney injury in asphyxiated newborns treated with therapeutic hypothermia. J Pediatr 2013;162:725-729.e1.

30. Carmody JB, Charlton JR. Short-term gestation, long-term risk: prematurity and chronic kidney disease. Pediatrics 2013;131:1168-79.

31. Goldstein SL, Devarajan P. Acute kidney injury in childhood: should we be worried about progression to CKD? Pediatr Nephrol 2011;26:509-22.

32. Boor P, Ostendorf T, Floege J. Renal fibrosis: novel insights into mechanisms and therapeutic targets. Nat Rev Nephrol 2010;6:643-56.

33. Moussad EE, Brigstock DR. Connective tissue growth factor: what's in a name? Mol Genet Metab 2000;71:276-92.

34. Grotendorst GR, Okochi H, Hayashi N. A novel transforming growth factor beta response element controls the expression of the connective tissue growth factor gene. Cell Growth Differ 1996;7:469-80. 
35. Dündar M, Kocak I, Culhaci N. Effects of long-term passive smoking on the diameter of glomeruli in rats: Histopathological evaluation. Nephrology (Carlton) 2004;9:53-7.

36. Huang LT, Chou HC, Lin CM, Yeh TF, Chen CM. Maternal nicotine exposure exacerbates neonatal hyperoxia-induced lung fibrosis in rats. Neonatology 2014;106:94-101.

37. Murrin LC, Ferrer JR, Zeng WY, Haley NJ. Nicotine administration to rats: methodological considerations. Life Sci 1987;40:1699-708.
38. Lichtensteiger W, Ribary U, Schlumpf M, Odermatt B, Widmer HR. Prenatal adverse effects of nicotine on the developing brain. Prog Brain Res 1988;73:137-57.

39. Ito $\mathrm{H}$, Yan $\mathrm{X}$, Nagata $\mathrm{N}$, et al. PGD2-CRTH2 pathway promotes tubulointerstitial fibrosis. J Am Soc Nephrol 2012;23:1797-809.

40. Kurus M, Ugras M, Esrefoglu M. Effect of resveratrol on tubular damage and interstitial fibrosis in kidneys of rats exposed to cigarette smoke. Toxicol Ind Health 2009;25:539-44. 